\title{
Design and Construction of a Digital Multichannel Analyzer for HPGe Detector Using Digital Signal Processing Technique
}

\author{
Dang Hong Ngoc Quy*, Pham Ngoc Tuan, Nguyen Nhi Dien \\ Nuclear Research Institute, DaLat, Viet Nam \\ Email: *danghongngocquy@gmail.com
}

How to cite this paper: Quy, D.H.N., Tuan, P.N. and Dien, N.N. (2019) Design and Construction of a Digital Multichannel Analyzer for HPGe Detector Using Digital Signal Processing Technique. Journal of Analytical Sciences, Methods and Instrumentation, 9, 22-29.

https://doi.org/10.4236/jasmi.2019.92003

Received: March 28, 2019

Accepted: June 2, 2019

Published: June 5, 2019

Copyright $\odot 2019$ by author(s) and Scientific Research Publishing Inc. This work is licensed under the Creative Commons Attribution International License (CC BY 4.0).

http://creativecommons.org/licenses/by/4.0/

c. (i) Open Access

\begin{abstract}
The outstanding advantage of digital signal processing (DSP) techniques and Field Programmable Gate Array (FPGA) technology is capable of improving the quality of the experimental measurements for nuclear radiation. In this article, a compact DMCA $8 \mathrm{~K}$ was designed and manufactured using DSP technique based on FPGA technology. In particular, the output of the preamplifier is completely processed by digital techniques which are obtained from the analog-to-digital converter (ADC) to calculate the baseline, DC offset, energy peaks, pile up, threshold discrimination and then the form of energy spectrum. The Spartan- 6 board is used as a hardware for the development of the digital multichannel analyzer (DMCA), which is equipped with the 14-bit AD6645 with 62.5 Msps sample rate. The application software for instrument control, data acquisition and data processing was written under $\mathrm{C}++$ builder via the RS-232 interface. The designed DMCA system has been tested with a HPGe detector using gamma sources of ${ }^{60} \mathrm{Co}$ and ${ }^{137} \mathrm{Cs}$ and a reference pulser.
\end{abstract}

\section{Keywords}

Field Programmable Gate Arrays, Digital Multichannel Analyzer, Integral Nonlinearity, Digital Signal Processing

\section{Introduction}

The task of electronics in systems of nuclear physics is to collect the electrical charge deposited in a radiation detector and to convert it into a digital code to be further analyzed by digital elements. In most cases, the significant quantities are the particle energy and the time of particle occurrence. The traditional spectros- 
copy systems for the particle detectors have been made of almost all analog parts constituting a chain of the preamplifier, shaping amplifier and peak sensing $\mathrm{ADC}$ configured as separate units. In the recent period, most of the radiation measurement systems designed at Dalat Nuclear Research Institute have been digitalized such as the FPGA-based MCA-8K [1]. However, the above-mentioned system only approaches DSP technique to obtain the exact peak and store to DPRAM. The goal of our design was to build a digital multi-channel analyzer system that would completely base on DSP techniques. This system has functioned as follows:

- Digital implementation of the shaping amplifier and peak sensing ADC,

- Charge sensitive preamplifier directly connected to the digitizer,

- Providing pulse height, time-stamp (16 ns) and optionally raw data,

- Pile-up rejection, baseline restoration, ballistic deficit correction,

- Best suited for high-resolution spectroscopy (HPGe and Si detectors).

For such purposes, a set of algorithms used in the digital spectrometric channel containing all necessary data acquisition elements have been developed.

\section{The Overall Design of DMCA System}

The block diagram of DMCA is shown in Figure 1 The DMCA is composed of $\mathrm{A} / \mathrm{D}$ converter, the CR and pole-zero cancellation, digital pulse processing (DPP) integrated into FPGA, RS-232 communication interface and power supply unit.

\subsection{A/D Converter}

The analog signal from particle detector is transferred to the high-speed sampling ADC for analog-to-digital conversion in real time into FIFO (First-in First-Out) within FPGA. In this design, 14-bitAD6645 is used as a waveform digitizer at a sampling rate of $62.5 \mathrm{MHz}$.

\subsection{CR Differentiator and Pole Zero Cancellation}

The output of collected data from FIFO is an exponential signal with long decay time $(50 \mu \mathrm{s})$ which is shortened to $3 \mu$ s by C-R differentiator. However; it creates an undershoot. A pole-zero cancellation (PZC) circuit is often applied to eliminate undershoot in many radiation detectors.

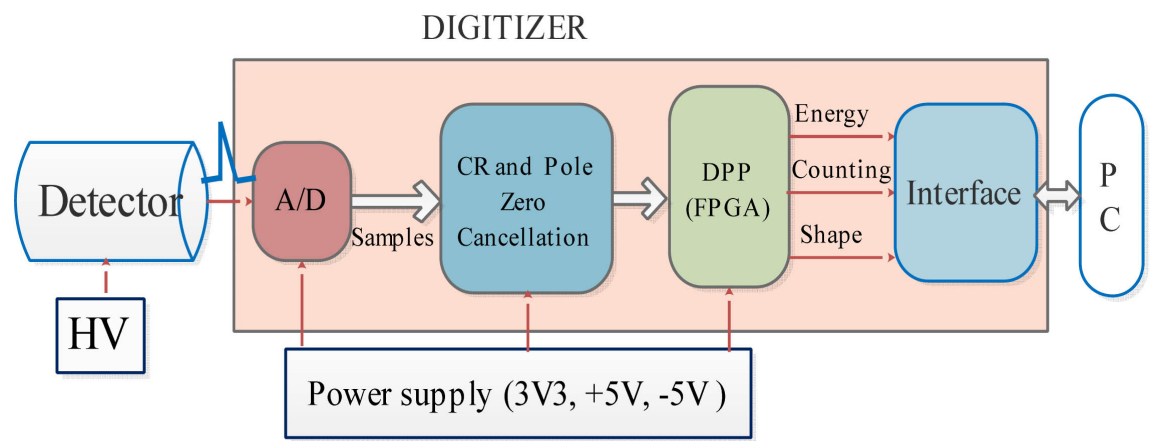

Figure 1. The block diagram of DMCA. 


\subsection{DPP for DMCA}

Figure 2 illustrates the DPP system consisting of the various component circuits. In particular, the $\mathrm{RC}-(\mathrm{CR})^{\mathrm{N}}$ filter identifies pulses, generates triggers and time-stamp. These triggers are also used to initialize all components in the system. The trapezoidal energy filter shapes the input signal to form a symmetrical shape of the trapezoid [2], restores the baseline and calculates the pulse height. The drift of the baseline mainly is due to respiration or patient movement, variations in temperature and bias in the instrumentation as well. In order to eliminate baseline drift, an efficient algorithm for baseline drift correction is implemented by digital averaging of the trapezoid wave in cases when the spectrometric channel is not busy. The accurate pulse height is obtained by subtracting the raw pulse height of the trapezoidal energy filter with the level of average baseline. At the same time, a fast trapezoidal filter is used for detecting the pile-up input pulse, eliminating pile-up pulses and improving energy resolution. Energy spectrum stored in the built-in RAM of DPP is transferred to the computer via RS-232 port under the controlling of small microprocessor named KPCSM6 supported from Xilinx [3]. The fabricated layout and front panel are illustrated in Figure 3.

\subsection{The Application Software}

For controlling of data acquisition, setting of parameters and interfacing with the computer, an application program was developed, achieving lots of functions including RS-232 serial communication, system parameter settings, spectrum display and spectrum data management, energy calibration, etc.

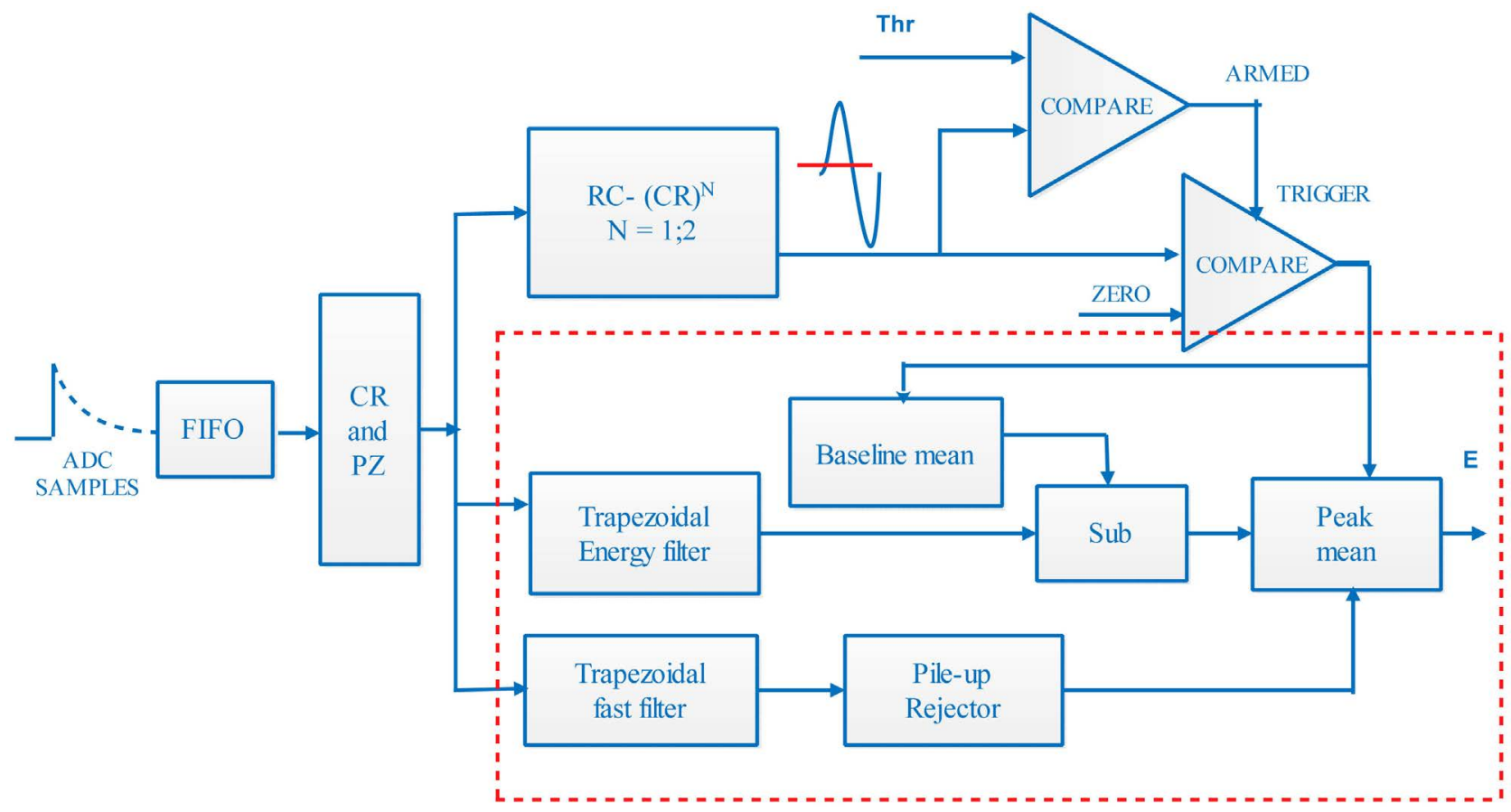

Figure 2. Main function blocks of the DPP. 

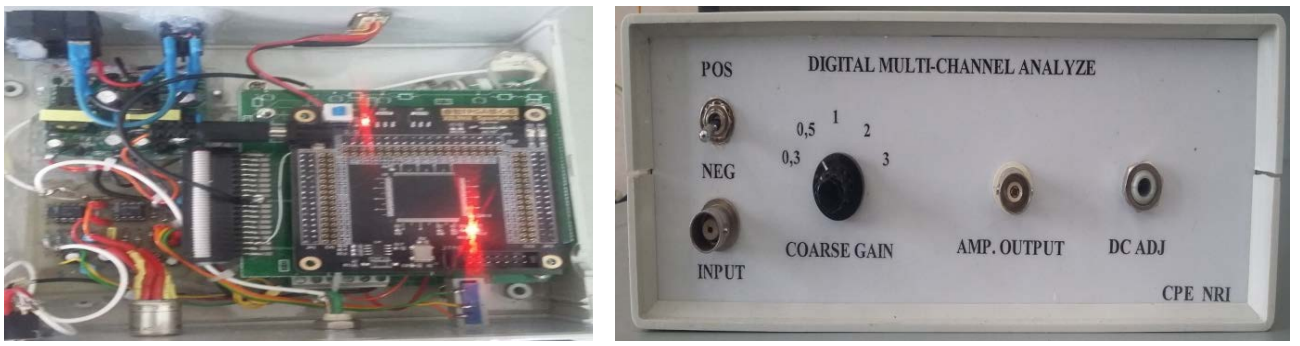

Figure 3. Layout of the DMCA on the left and its front on the right.

\section{Experimental Measurements and Results}

\subsection{Determination of Integral Nonlinearity of DMCA}

With DMCA system, precise energy calibration of a spectrum depends on the linearity of the system. To test the integral nonlinearity of DMCA, an experimental configuration is performed with reference pulser DB-2 generating a positive nuclear tail pulse with the rise time of $100 \mathrm{~ns}$ and fall time of $50 \mu \mathrm{s}$. Changing the incremental steps from 0 to $1100 \mathrm{mV}$, with the $44 \mathrm{mV}$ step up. The corresponding channel-voltage value pairs are listed in Table 1.

Figure 4 reveals the experimental fitting function of $y=0.10708 x+214.18442$, where $\mathrm{x}$ is the amplitude of the input signal, $\mathrm{y}$ is the estimated channel. The coefficient of determination $\mathrm{R}^{2}=0.99935$. Replacing $X_{\mathrm{i}}=(197 \div 866.8 \mathrm{mV})$ with i $\in[1,44]$, then 44 values of $Y_{\mathrm{i}}$ is obtained. Therefore, $\Delta Y_{\max }=\left(Y_{r}-Y_{i}\right)_{\max }=$ 22.354 and $Y_{\max }=7777$. According to reference (ORTEC, CAMAC ADCs and Memories), integral nonlinearities are calculated as follows:

$$
I N L_{D M C A}=\frac{\Delta Y_{\max }}{Y_{\max }} \times 100 \%=\frac{22.354}{7777}=0.28 \%
$$

\subsection{The Effects of Input Rise Time on Peak Shape and FWHM}

Figure 5 shows the comparison of the peak of ${ }^{137} \mathrm{Cs}$ from HPGe detector with different input rise times. The lowest amplitude peak is for input rise time of 20 $\mathrm{ns}(\mathrm{FWHM}=7 \mathrm{keV})$, the center peak is for input rise time of $50 \mathrm{~ns}(\mathrm{FWHM}=5$ $\mathrm{keV})$ and the highest peak is for input rise time of $100 \mathrm{~ns}(\mathrm{FWHM}=4 \mathrm{keV})$.

\subsection{The Effects of Pile-Up Rejection on Energy Spectrum}

Figure 6 shows the effects of pil-up mode on Compton background in gamma spectroscopy. To optimize for a Compton suppression detector system, pile-up mode is added. When being activated, it provides more-clear photopeak from a ${ }^{137} \mathrm{Cs}$ and ${ }^{60} \mathrm{Co}$ source above the Compton continuum.

\subsection{The Effects of Input Count Rate on the Peak Position}

Peak channel of the generated pulse resolved by the DMCA is shown in Table 2 and presented in Figure 7. The effects of input count rate on gamma-ray peak shift have been done using DB-2 pulse generator. An experimental arrangement is performed with rise time $=100 \mathrm{~ns}$, fall time $=10 \mu$ s, time measurement $=100 \mathrm{~s}$. 


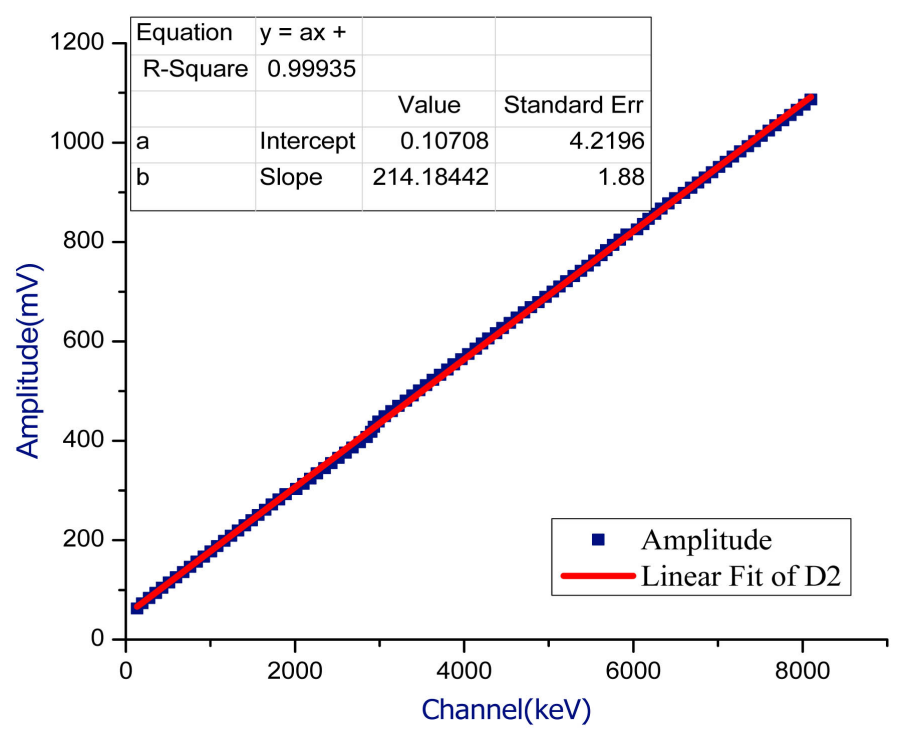

Figure 4. Integral nonlinearity of DMCA.

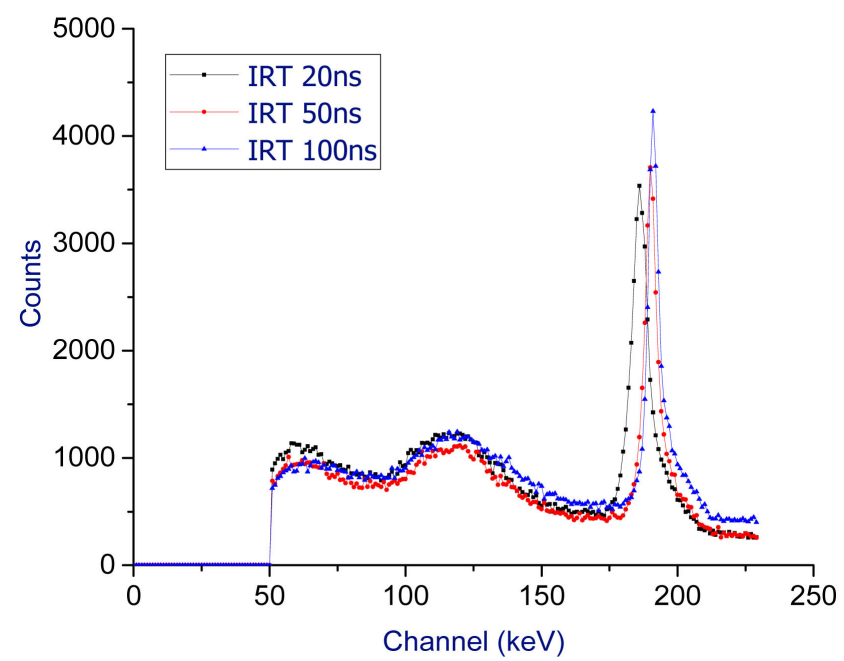

Figure 5. Comparison of spectrum peak shapes with different shaping times.

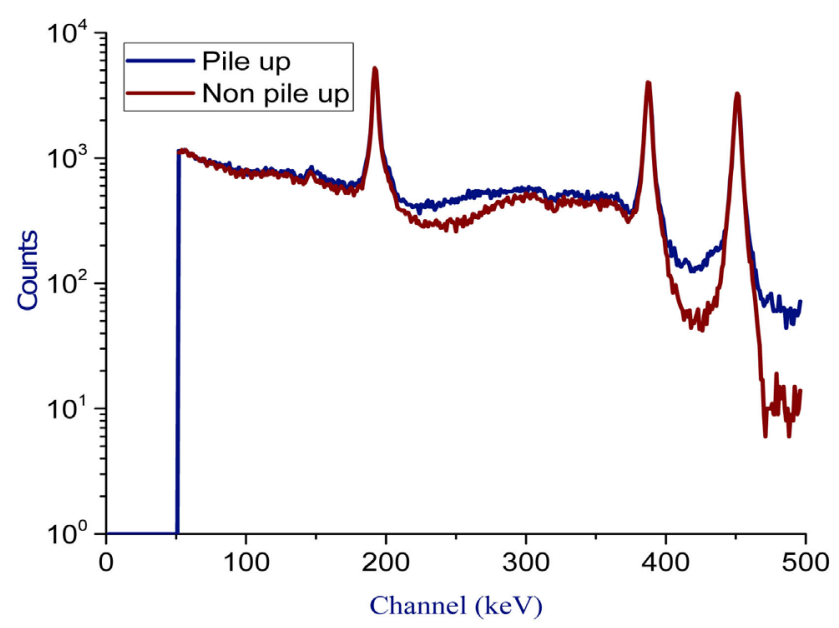

Figure 6. The effects of pile-up mode on the energy spectrum. 
Table 1. Results of integral nonlinearity of the DMCA.

\begin{tabular}{|c|c|c|c|c|c|c|c|c|c|}
\hline No. & $X(\mathrm{mV})$ & $Y_{r}$ & $Y_{i}$ & $\Delta Y_{\max }$ & No. & $X(\mathrm{mV})$ & $Y_{r}$ & $Y_{i}$ & $\Delta Y_{\max }$ \\
\hline 1 & 197 & 48 & 219.354 & -22.354 & 23 & 630.4 & 3912 & 635.538 & -5.138 \\
\hline 2 & 216.7 & 109 & 225.925 & -9.225 & 24 & 650.1 & 4095 & 655.249 & -5.149 \\
\hline 3 & 236.4 & 263 & 242.512 & -6.112 & 25 & 669.8 & 4271 & 674.205 & -4.405 \\
\hline 4 & 256.1 & 429 & 260.391 & -4.291 & 26 & 689.5 & 4462 & 694.778 & -5.278 \\
\hline 5 & 275.8 & 615 & 280.425 & -4.625 & 27 & 709.2 & 4644 & 714.380 & -5.180 \\
\hline 6 & 295.5 & 759 & 295.935 & -0.435 & 28 & 728.9 & 4823 & 733.660 & -4.760 \\
\hline 7 & 315.2 & 941 & 315.538 & -0.338 & 29 & 748.6 & 4947 & 747.016 & 1.584 \\
\hline 8 & 344.9 & 1118 & 334.602 & 0.298 & 30 & 768.3 & 5130 & 766.726 & 1.574 \\
\hline 9 & 354.6 & 1297 & 353.882 & 0.718 & 31 & 788 & 5230 & 777.497 & 10.503 \\
\hline 10 & 374.3 & 1458 & 371.223 & 3.077 & 32 & 807.7 & 5476 & 803.993 & 3.707 \\
\hline 11 & 394 & 1655 & 392.441 & 1.559 & 33 & 827.4 & 5671 & 824.996 & 2.404 \\
\hline 12 & 413.7 & 1795 & 407.520 & 6.180 & 34 & 847.1 & 5858 & 845.138 & 1.962 \\
\hline 13 & 433.4 & 1963 & 425.615 & 7.785 & 35 & 866.8 & 6105 & 871.742 & -4.942 \\
\hline 14 & 453.1 & 2161 & 446.941 & 6.159 & 36 & 866.5 & 6308 & 893.606 & -7.106 \\
\hline 15 & 472.8 & 2330 & 465.144 & 7.656 & 37 & 906.2 & 6517 & 916.117 & -9.917 \\
\hline 16 & 492.5 & 2498 & 483.239 & 9.261 & 38 & 925.9 & 6696 & 935.397 & -9.497 \\
\hline 17 & 512.2 & 2698 & 504.781 & 7.419 & 39 & 945.6 & 6880 & 955.215 & -9.615 \\
\hline 18 & 531.9 & 2906 & 527.184 & 4.716 & 40 & 965.3 & 7058 & 974.387 & -9.087 \\
\hline 19 & 551.6 & 3183 & 557.019 & -5.419 & 41 & 985 & 7239 & 993.883 & -8.883 \\
\hline 20 & 571.3 & 3375 & 577.699 & -6.399 & 42 & 1004.7 & 7408 & 1012.085 & -7.385 \\
\hline 21 & 591 & 3551 & 596.656 & -5.656 & 43 & 1024.4 & 7595 & 1032.227 & -7.827 \\
\hline 22 & 610.7 & 3727 & 615.612 & -4.912 & 44 & 1044.1 & 7777 & 1051.830 & -7.730 \\
\hline
\end{tabular}

Table 2. Count-rate performance of DMCA $8 \mathrm{~K}$.

\begin{tabular}{|c|c|c|c|c|c|c|c|c|}
\hline \multirow{2}{*}{$\begin{array}{l}\text { Count } \\
\text { rate } \\
\text { (Kcps) }\end{array}$} & \multicolumn{2}{|c|}{$1 \mu \mathrm{s}$} & \multicolumn{2}{|c|}{$2 \mu \mathrm{s}$} & \multicolumn{2}{|c|}{$3 \mu \mathrm{s}$} & \multicolumn{2}{|c|}{$4 \mu \mathrm{s}$} \\
\hline & $\begin{array}{c}\text { Peak } \\
\text { channel (ch) }\end{array}$ & $\begin{array}{c}\text { Peak } \\
\text { shift (\%) }\end{array}$ & $\begin{array}{c}\text { Peak } \\
\text { channel }\end{array}$ & $\begin{array}{l}\text { Peak } \\
\text { shift }\end{array}$ & $\begin{array}{c}\text { Peak } \\
\text { channel }\end{array}$ & $\begin{array}{l}\text { Peak } \\
\text { shift }\end{array}$ & $\begin{array}{c}\text { Peak } \\
\text { channel }\end{array}$ & $\begin{array}{l}\text { Peak } \\
\text { shift }\end{array}$ \\
\hline 0.1 & 3485 & 0 & 3185 & 0 & 2903 & 0 & 2785 & 0 \\
\hline 0.5 & 3485 & 0 & 3185 & 0 & 2903 & 0 & 2785 & 0 \\
\hline 0.7 & 3484 & 0.02 & 3184 & 0.03 & 2901 & 0.07 & 2784 & 0.04 \\
\hline 10 & 3478 & 0.2 & 3148 & 1.2 & 2892 & 0.3 & 2775 & 0.4 \\
\hline 15 & 3466 & 0.5 & 3132 & 1.7 & 2876 & 0.9 & 2758 & 0.9 \\
\hline 20 & 3439 & 1.3 & 3098 & 2.7 & 2838 & 2.2 & 2722 & 2.2 \\
\hline 30 & 3375 & 2.3 & 3021 & 4 & 2757 & 4.7 & 2641 & 4.8 \\
\hline 40 & 3285 & 5.2 & 2910 & 7.1 & 2644 & 8.1 & 2530 & 8.2 \\
\hline 50 & 3220 & 6.3 & 2829 & 8.7 & 2564 & 9.6 & 2453 & 9.8 \\
\hline
\end{tabular}


Figure 7 shows a slight descending trend with increasing ICR for the position of peaks with different shaping time $(1 ; 2 ; 3$ and $4 \mu \mathrm{s})$. This means that with the increase of count rate, all the energy peaks shifted to lower energies.

\subsection{Energy Resolution (FWHM)}

The energy spectra collected from the ${ }^{60} \mathrm{Co}$ are shown in Figure 8 with the percent FWHM energy resolution of each discernible peak labeled. In particular, FWHM of ${ }^{60} \mathrm{Co}$ at 1173 is $5 \mathrm{keV}$ comparing to $4 \mathrm{keV}$ with ORTECT's DSPEC jr.

\section{Conclusions}

This work carried out a design and construction of a compact DMCA-8K based on FPGA for high resolution gamma spectroscopy. This article also conducts an observation on the performance and quality of this system such as input count rate, FWHM, peak shape, peak position, pile up rejection, integral nonlinearity. Main technical characteristics are as follows:

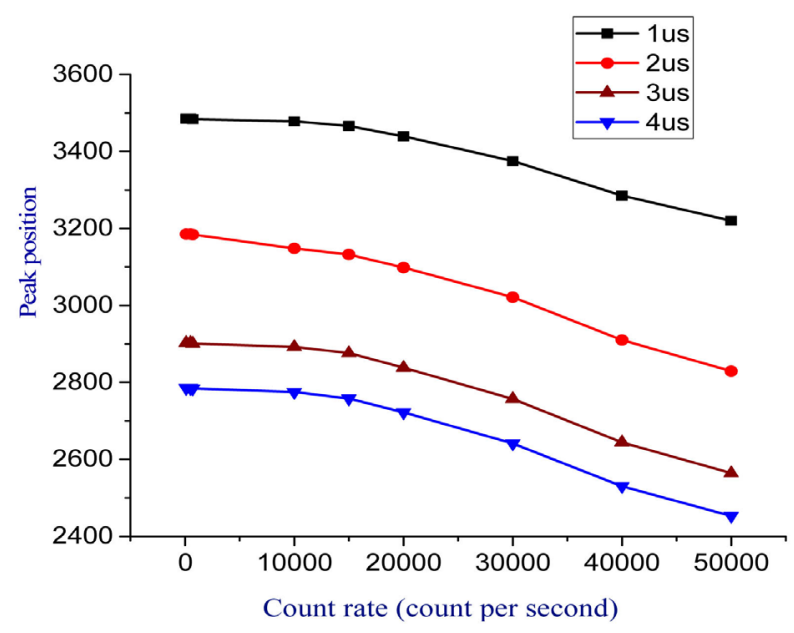

Figure 7. The effects of various input count rate on peak position.

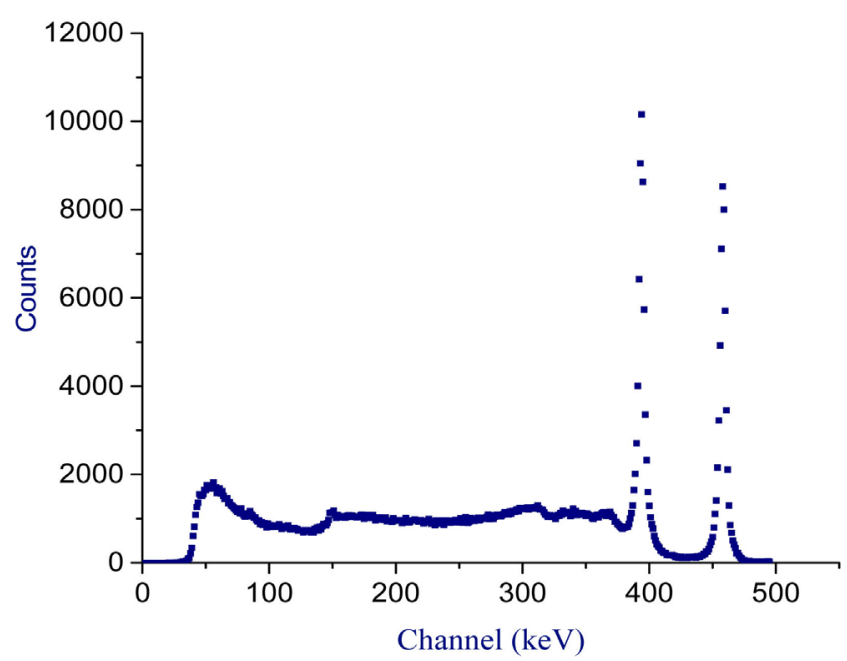

Figure 8. $\gamma$-ray spectrum of ${ }^{60} \mathrm{Co}$ measured by DMCA. 
- ADC conversion bit: 14-bit, sampling rate: $62.5 \mathrm{MHz}$

- Resolution: 8192 channels,

- The integral nonlinearity: $0.23 \%$,

- Maximum count capacity per channel: $2^{32}-1$,

- Shaping time: $1 ; 2 ; 4 ; 6$ and $12 \mu$ sy software,

- Course gain: $0.3 ; 0.5 ; 1 ; 2$ and 3 ,

- DB9 connector for preamplifier power supply

- On-line pile-up rejection,

- The input receiving positive, unipolar pulse peak amplitude from 0 to $1.1 \mathrm{~V}$,

- Data acquisition program MCA DAQ has written in CBuilder ++ 6.0, self-executing under Windows XP environment.

\section{Conflicts of Interest}

The authors declare no conflicts of interest regarding the publication of this paper.

\section{References}

[1] Lanh, D., Son, P.N. and Son, N.A. (2014) In-House Development of an FPGA-Based MCA8K for the Gamma-Ray Spectrometer. SpringerPlus, 3, 665. https://doi.org/10.1186/2193-1801-3-665

[2] Jordanov, V.T. and Knoll, G.F. (1994) Digital Synthesis of Pulse Shapes in Real Time for High Resolution Radiation Spectroscopy. Nuclear Instruments and Methods in Physics Research Section A: Accelerators, Spectrometers, Detectors and Associated Equipment, 345, 337-345. https://doi.org/10.1016/0168-9002(94)91011-1

[3] XILINX (2011) PicoBlaze 8-Bit Embedded Microcontroller User Guide. 